\title{
Biogen and immune disease
}

The immune system provides a protective mechanism through which the body defends itself against invading organisms. For centuries, scientists and physicians have sought ways to increase the body's ability to resist these pathogens, as well as to solve the riddles of diseases that occur when the body turns its immune system against itself. While there have been many successes, the problems of infectious and autoimmune diseases remain among the most profound unsolved questions in medicine.

The revolution in genetic engineering has provided important new approaches to solving these problems, through intensive study in both academic and pharmaceutical laboratories worldwide, including Biogen, which has a long and distinguished history of research into immune disease. Founded in 1978 by an international group of distinguished scientists, two of Biogen's early discoveries are among the world's leading pharmaceutical products. They are INTRON ${ }^{\circledR}$ A alpha interferon, marketed throughout the world by Schering-Plough Corporation, for treatment of hepatitis B and C and certain cancers, and the hepatitis B vaccines, sold by SmithKline Beecham and Merck. In 1996, Biogen received licensure to market AVONEX ${ }^{\circledR}$ (Interferon beta-1a), which has become the world's leading treatment for relapsing forms of the autoimmune disease multiple sclerosis. Biogen has maintained its strength in this arena of biology, and is investigating multiple molecules and molecular pathways relevant to autoimmune and allergic diseases. These include members of the TNF family, such as CD40 ligand and lymphotoxin beta, the integrins, particularly VLA4 and its interaction with VCAM1, the CD2/LFA3 pathway, and, of course, the interferons.

Biogen believes that continued fundamental research in immunology will be of central importance to our understanding of human disease. Few pathologies are unaccompanied by inflammatory and immune responses. The rapid pace of advances in this area, combined with novel technologies for drug discovery and the sequencing of the human genome, promise to produce an unprecedented increase in the pace of drug discovery in the first years of the new millennium. As such, we are proud to sponsor this supplement, which showcases some of the key advances made in the last year in our understanding of both innate and acquired immune responses.

Joseph M. Davie, MD PhD, Senior Vice President-Research

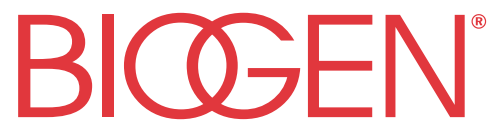

Winner of the 1998 National Medal of Technology ${ }^{\circledR}$ 